\title{
Rhinosporidiosis: Endoscopic Excision and Review of Literature
}

\section{Manish Gupta}

\section{ABSTRACT}

Objective: To show that endoscopic excision of rhinosporidiosis carries less risk of recurrence.

Materials and methods: Five consecutive patients of rhinosporidiosis were placed for endoscopic excision. The various sized mass were excised completely with safe margin under better illumination, magnification and hemostasis using $30^{\circ}$ endoscope with camera and monitor. All the patients were started on dapsone therapy after surgery for 3 months.

Results: There was no recurrence seen on an average 1 year follow-up.

Conclusion: The nasal endoscope plays an important role in localization of site of origin and ensures complete excision with no complications and recurrence. Thus, endoscopic technique of rhinosporidiosis excision is recommended.

Keywords: Rhinosporidiosis, Endoscopic surgery, Nose.

How to cite this article: Gupta M. Rhinosporidiosis: Endoscopic Excision and Review of Literature. Clin Rhinol Int J 2012;5(1): 32-34.

Source of support: Nil

Conflict of interest: None declared

\section{INTRODUCTION}

Rhinosporidiosis is a chronic disease caused by fungus Rhinosporidium seeberi. ${ }^{1}$ It typically involves mucosal surfaces, most frequently the nasal mucosa ${ }^{1}$ and the adjacent skin. It occurs worldwide, although it is endemic in South Asia with highest incidence from India and Sri Lanka. ${ }^{1}$ Surgery is the mainstay of treatment, but there is frequent recurrence, ${ }^{2}$ although in endemic areas it is not easy to establish whether recurrent lesions are due to relapse or re-infection.

We hereby discuss the endoscopic technique of rhinosporidiosis excision and its advantages, used in five consecutive patients in nonendemic area of North India.

\section{MATERIALS AND METHODS}

Five consecutive patients of rhinosporidiosis (Table 1), presenting with nasal mass in our outpatient department were considered for this prospective study. Routine blood investigations, such as hemoglobin, total and differential leukocyte counts, erythrocyte sedimentation rate, bleeding and clotting time were undertaken. All patients were taken up for endoscopic excision of mass under local anesthesia. The nasal masses were excised completely along with part

\section{Table 1: Clinical presentation}

\begin{tabular}{lllc}
$\begin{array}{l}\text { S. } \\
\text { No. }\end{array}$ & Site of origin & Size $(\mathrm{cm})$ & $\begin{array}{c}\text { No recurrence } \\
\text { (in months) }\end{array}$ \\
\hline 1. & Left inferior turbinate & $3 \times 1 \times 0.3$ & 20 \\
2. & Left inferior turbinate & $3.5 \times 1.2 \times 0.5$ & 17 \\
3. & Left inferior meatus & $5.2 \times 1.2 \times 0.4$ & 16 \\
4. & Right side nasal septum & $2 \times 0.7 \times 0.4$ & 12 \\
5. & Right middle turbinate & $3.2 \times 0.8 \times 0.4$ & 10 \\
\hline
\end{tabular}

of surrounding mucosa. After excision, the attachment site and the mucosa around were electrocoagulated taking due care not to damage the surrounding structures. Nasal cavity was cleaned and medicated ribbon gauze packed. The pack was removed the next day. The patient was started on dapsone therapy after surgery for 3 months.

\section{REPRESENTATIVE CASE}

A 22-year-old male laborer, resident of Nepal reported in our outpatient department with complaint of left sided nasal obstruction. He gave history of a mass protruding out of nostril on sneezing. History of nasal discharge with occasional bleeding was present. There was no history of any medical or surgical treatment received in past for the same. Clinical examination of left side nasal cavity revealed polypoidal, irregular, reddish friable mass with white dots all over its surface (Fig. 1). On diagnostic nasal endoscopy the mass was found originating from inferior meatus and hanging freely into nasopharynx posteriorly (Fig. 2). The noncontrast computed tomogram nose and paranasal sinuses revealed no signs of sinus involvement or of bone destruction. After endoscopic excision the mass was sent for histopathological examination. Grossly, grey brown tissue measuring $5.2 \times 1.2 \times 0.4 \mathrm{~cm}$ was seen. The hematoxylin- eosin stained sections showed respiratory epithelium with deep invaginations forming pseudocysts. Numerous sporangia's at different stages of development were seen (Figs 3 and 4). The submucosa was found to be infiltrated by mixed inflammatory cells. The patient was managed by surgical technique already described and until today there is no recurrence after 1 year of follow-up.

\section{DISCUSSION}

Rhinosporidiosis is thought to be caused by fungus Rhinosporidium seeberi. The taxonomy of causative agent is unclear. Though most microbiologists consider it as 


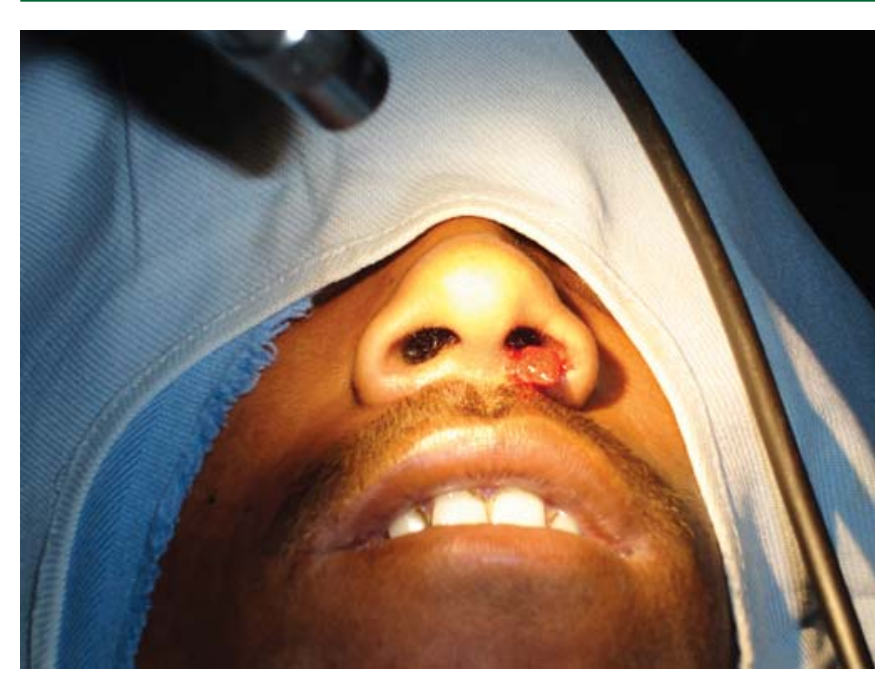

Fig. 1: Clinical photograph showing rhinosporidiosis mass protruding out of left nostril

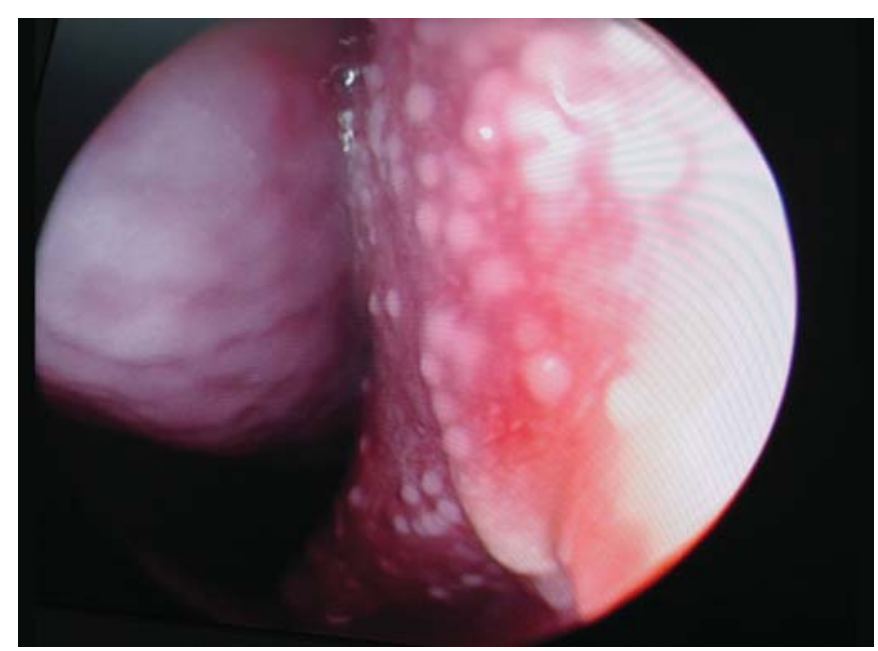

Fig. 2: Endoscopic picture showing rhinosporidiosis mass in left inferior meatus with multiple white dots on surface

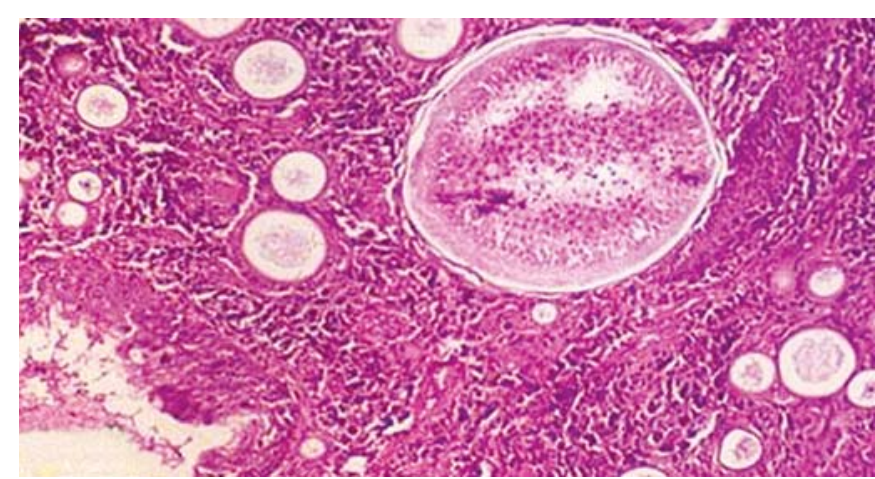

Fig. 3: Microscopic picture at low power after hematoxylin and eosin staining showing numerous spherules of varying sizes typical of rhinosporidiosis

fungus just because of its property to be stained by fungal stains; the uncertainty arises from the difficulty to isolate and to grow the microorganism in culture. Ahluwalia ${ }^{3}$ hypothesized that causative agent was not a fungus but a

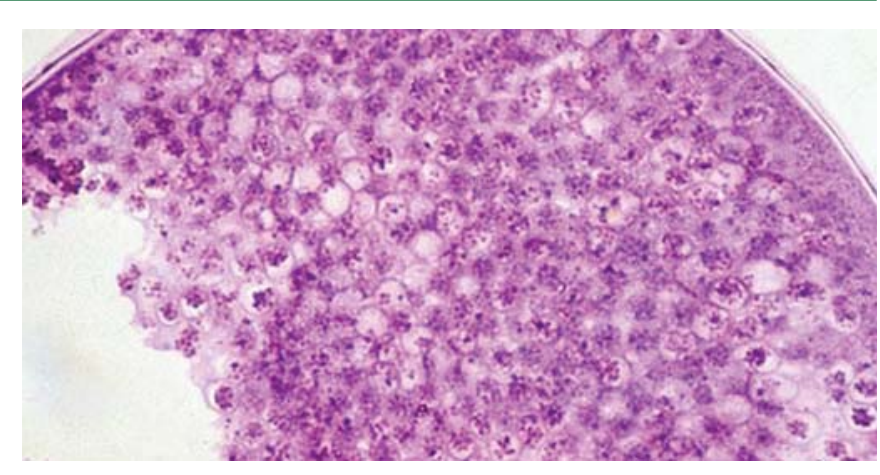

Fig. 4: Microscopic picture at high power showing mature spherule with endospores typical of rhinosporidiosis

prokaryotic cyanobacterium Microcystis aeruginosa, based on the findings that this organism was isolated from both clinical specimens of patient and the pond water samples where they had bathed.

The presumed mode of infection from natural aquatic habitat of Rhinosporidium seeberi is through traumatized nasal epithelium ${ }^{2}$ (transepithelial infection). The occurrence of rhinosporidiosis in river sand workers in India and Sri Lanka is partially relevant to such a mode of infection through abrasions caused by sand particles with the pathogen in putative habitat ground water. Spillage of endospores from polyp after trauma or surgery is thought to be followed by autoinoculation through the adjacent epithelium. ${ }^{2}$

The cases generally involve anterior nares, inferior turbinate, septum, floor, nasopharynx, larynx and soft palate. Urethral, vaginal, rectal, conjunctival mucosa, skin and lacrimal sac may also be involved rarely. ${ }^{1}$ In our series of five patients two had mass arising from inferior turbinate, one each from inferior meatus, septum and middle turbinate (Table 1).

Epistaxis is often the only symptom, but in the early stages there is a viscid nasal discharge with irritation and partial obstruction. ${ }^{1}$ Clinically, rhinosporidiosis usually presents as papillomatous and polypoid lesions. ${ }^{1}$ These lesions are soft, highly vascular, sessile or pedunculated and the grayish under-surface studded with white dots resembles a strawberry. Disease progresses with the local replication of $R$. seeberi and associated hyperplastic growth of host tissue and an inflammatory response. ${ }^{4}$

Differential diagnosis includes nasal polyp, hemangioma, malignancy and Coccidioides immitis. Diagnosis of rhinosporidiosis rests on the finding of the typical structures of Rhinosporidium seeberi in the tissues. The organisms stain with the periodic acid schiff (PAS) reaction at all stages. ${ }^{4}$ The morphologic characteristics of Rhinosporidium seeberi resemble those of Coccidioides immitis. Both organisms have mature stages that consist of large, thick-walled, spherical structures containing smaller 
daughter cells (endospores). Cytological criteria for diagnosis of rhinosporidiosis are based on the presence of endospores with diameter of 5 to $10 \mu \mathrm{m}$ and sporangia of diameter 50 to $1000 \mu \mathrm{m}$. These findings made it easy to differentiate rhinosporidiosis from the more common nasal mycoses, such as Coccidioides immitis which has diameter 2 to $4 \mu \mathrm{m}$ and 20 to $80 \mu \mathrm{m}$ of endospores and sporangia respectively. 4

Complications of the disease include extremely rare lifethreatening dissemination, osteolytic lesions of the bone, ${ }^{5}$ secondary bacterial infection and recurrence. ${ }^{4}$ The possible causes for the recurrence of this disease include incomplete removal of mass or continued exposure to the infective environment. Microscopic study of the biopsy in recurrent cases had shown extreme overcrowding of the sporangia, thereby suggesting that the traumatic conditions caused by repeated surgery may act as a predisposing factor for recurrence. Similarly, variation in the virulence of the pathogen $v s$ the lesions produced or the altered immunological status of the host due to subsequent infections may also be responsible for this condition. As many facets of rhinosporidiosis, such as the life cycle of the pathogen, mode of its transmission and the events in pathogenesis are still not clear, no precise reason could be attributed for its recurrence.

Surgical excision with cautery of the base of lesion is known to reduce the risk of recurrence. ${ }^{2}$ Various medical therapies including antifungal have been tried but dapsone (4, 4 diaminodiphenylsulfone) is the only drug claimed to have antirhinosporidial effect. It arrests the maturation of sporangia and promotes fibrosis in the stroma, when used as adjunct to surgery. ${ }^{6}$ But only antimicrobial therapy is ineffective, and the disease may recur after months or years. ${ }^{4}$

The use of endoscope helps in removing the entire mass which can not be seen by anterior rhinoscopy or conventional surgery. Endoscope gives better illumination and helps in removing the entire pathology precisely with minimal exposure, limited manipulation and least resection of normal surrounding mucosa. This technique causes fewer traumas to the septum and turbinates, thus reducing the postoperative complications like hemorrhage and synechiae formation. In a similar case report of naso-oropharyngeal rhinosporidiosis excision under endoscopic guidance the author had stated that only endoscopic visualization could reveal the attachment of mass at posterior end of the septum and en-bloc removal could be done only after endoscopic guided cauterization of the base. ${ }^{7}$

In a recent series of rhinosporidiosis treatment, KTP532 laser was used. ${ }^{8}$ The advent of the sinus endoscope has facilitated the clearance of smaller granuloma but bleeding from the larger granuloma can impair vision and pose difficulties. The introduction of the surgical laser for fulguration and vaporization of this granuloma would be a logical step, as one would expect this to reduce bleeding and also improve vision with the endoscope. An additional advantage of using laser is minimum physical contact with the fungal granuloma, thus surrounding tissue seedling is minimal.

\section{REFERENCES}

1. Weir N. Golding-Wood DG. Infective rhinitis and sinusitis. In: Mackay IS, Bull TR (Eds) Scott-Brown's Otolaryngology Butterworth-Heinemann, 1997;4(6):39-40.

2. Das A, Das AK. Endoscopic excision of recurrent rhinosporidiosis. MJAFI 2008;64:76-77.

3. Ahluwalia KB. Culture of the organism that causes rhinosporidiosis. J Laryngol Otol 1999;113:523-28.

4. Al-Shehry A, Hussein MR. Rhinosporidiosis of the nose in the southern region of the Kingdom of Saudi Arabia. J Oral Maxillofac Pathol 2007;11:83-85.

5. Sathyanarayana C. Rhinosporidiosis. Acta Otolaryngologica 1960;51:348-66.

6. Venkateswaran S, Date A, Job A, Mathan M. Light and electron microscopic findings in rhinosporidiosis after dapsone therapy. Trop Med Int Health 1997;2:1128-32.

7. Sonkhya N, Singhal P, Mishra P. Naso-oropharyngeal rhinosporidiosis: Endoscopic removal. IJOHNS 2005;57: 354-56.

8. Kameshwaran M, Anand Kumar RS, Sathiya M, Raghunandhan S, Jacob J. KTP-532 laser in the management of rhinosporidiosis. IJOHNS 2005;57:298-300.

\section{ABOUT THE AUTHOR}

\section{Manish Gupta}

Assistant Professor, Department of ENT, Gian Sagar Medical College and Hospital, Patiala, Punjab, India

\section{CORRESPONDING AUTHOR}

Manish Gupta, Assistant Professor, Department of ENT, 1156-C Type V Flats, Government Medical College and Hospital Campus, Sector 32-B, Chandigarh, India, Phone: 9915025819, 0172-2622117 e-mail: manishgupta1217@gmail.com 